\title{
As aves da Reserva Florestal do Morro Grande (Cotia, SP)
}

\author{
Pedro F. Develey ${ }^{1}$ \& Alexandre Camargo Martensen ${ }^{2}$
}

Biota Neotropica v6 (n2)-http:/www.biotaneotropica.org.br/v6n2/pt/abstract?article+bn00706022006

\author{
Recebido em 11/8/2004 \\ Versão reformulada recebida em 21/3/2005 \\ Publicado em 01/05/2006
}

${ }^{1}$ Birdlife International - Programa do Brasil, Rua Fernão Dias 219, Conjunto 2, 05427-010 São Paulo, SP (autor para correspondência: pdeveley@uol.com.br)

${ }^{2}$ Departamento de Ecologia, Instituto de Biociências, Universidade de São Paulo, Rua do Matão, 321, travessa 14, 05509-900 São Paulo, SP

\begin{abstract}
Develey, P.F. and Martensen, A.C. Birds of Morro Grande Reserve (Cotia, SP). Biota Neotrop. May/Aug 2006 vol. 6 no. 2 , http://www.biotaneotropica.org.br/v6n2/pt/abstract?article+bn00706022006. ISSN 1676-0603

A total of 198 bird species was recorded in the Morro Grande Forest Reserve. The survey was conducted between 2001 and 2003 using qualitative and quantitative (mist-nets and point counts) methods. The number of species recorded with the quantitative methods was lower if compared with the total number of species registered in the reserve, with 98 species recorded with point counts and 46 captured with mist nets. In spite of this difference, both methods detected similar patterns of species distribution, showing a huge heterogeneity in the bird community along the reserve, with the main differences between the mature forest, richer and with several exclusive species, and secondary forests. However, even among secondary forest plots differences could be seen. Besides the large size of the Reserve and the fact that it is well linked with the continuous forest of the Serra de Paranapiacaba, several species have already disappeared from the Reserve. These are mainly species under a high hunting pressure and the large canopy frugivores. Even though, the Morro Grande Reserve harbors a high diversity of species, with several endemic and threatened species. The conservation and restoration of Morro Grande forests represent important actions for bird conservation in the Atlantic forest.
\end{abstract}

Key words: birds, Atlantic forest, secondary forest, mature forest, mist-nets, point counts

\section{Resumo}

Develey, P.F. and Martensen, A.C. As aves da Reserva Florestal do Morro Grande Cotia, SP). Biota Neotrop. May/Aug 2006 vol. 6 no. 2, http://www.biotaneotropica.org.br/v6n2/pt/abstract?article+bn00706022006. ISSN 1676-0603

Um total de 198 espécies de aves foi registrado na Reserva Florestal do Morro Grande. O levantamento foi realizado entre os anos de 2001 e 2003, utilizando-se métodos de amostragem qualitativos e quantitativos (redes de neblina e pontos fixos). O número de espécies registrado através dos métodos quantitativos foi menor do que o número total de aves presentes na Reserva, sendo 98 espécies registradas nos pontos fixos e 46 nas redes de neblina. Apesar das diferenças, os dois métodos se mostraram eficientes na detecção de padrões de distribuição das aves. Existe uma grande heterogeneidade da avifauna na Reserva, sendo que as diferenças mais marcantes são entre os trechos de matas primárias e secundárias. As matas primárias são mais ricas e apresentam muitas espécies exclusivas. No entanto, mesmo entre os trechos de mata secundária também existem diferenças. Apesar de ser uma grande área de mata contígua às matas da Serra de Paranapiacaba, muitas aves parecem já ter desaparecido da Reserva, como as sensíveis à caça e os frugívoros de dossel. Mesmo assim a Reserva do Morro Grande abriga uma alta diversidade de espécies, com muitas aves endêmicas e ameaçadas. A manutenção e recuperação de suas matas representam importantes ações para a conservação das aves da Mata Atlântica.

Palavras-chave: aves, Mata Atlântica, mata secundária, mata primária, redes de neblina e ponto fixo 


\section{Introdução}

A Mata Atlântica é conhecida mundialmente pela sua imensa biodiversidade, número de espécies endêmicas e grau de ameaça (Myers et al. 2000). Cobria originalmente cerca de 1.350.000 km² (SOS Mata Atlântica/INPE 2002), numa faixa paralela à costa entre os Estados do Rio Grande do Norte e Rio Grande do Sul (S.M.A. 1996). Nas regiões sul e sudeste essa floresta se estendia pelo interior, chegando até a província de Misiones, no norte da Argentina. Desde o início da colonização portuguesa foi o bioma brasileiro que mais sofreu alterações provocadas pela ocupação humana, apresentando atualmente menos de 8\% da área florestal original (SOS Mata Atlântica/INPE 2002). Grande parte destes remanescentes está distribuída em diversos pequenos fragmentos (Metzger 2000) e é composta por florestas secundárias em diferentes graus de regeneração (Viana \& Tabanez 1996).

Apesar deste longo histórico de alterações, ainda abriga uma comunidade de aves extremamente rica e diversa, com um total de aproximadamente 700 espécies (Goerck 1997). Estas possuem distribuição bastante restrita (Stotz et al. 1996), possibilitando a existência de um grande número de espécies, além de uma das maiores concentrações de endemismos do mundo (Goerck 1997). Aproximadamente 200 espécies são endêmicas a este bioma, e destas, apenas 8,5\% ocorrem em habitats alterados (Goerck 1997), de modo que várias espécies são muito sensíveis a distúrbios ambientais. A contribuição das matas secundárias para a manutenção destas espécies ainda é pouco conhecida. Aleixo (1999) e Protomastro (2001) demonstraram que matas secundárias podem abrigar uma diversidade de aves similar às matas primárias, no entanto as espécies mais sensíveis desaparecem ou tornam-se raras.

O avançado processo de substituição de florestas por ambientes antropizados, associado ao fato de que muitas das espécies não ocorrem em habitats perturbados ou nas florestas secundárias, colocam o grupo como um dos mais ameaçados do planeta (Brooks \& Balmford 1996).

Esse cenário extremamente preocupante em relação à conservação da Mata Atlântica e de sua avifauna, faz urgente a tomada de ações concretas visando a manutenção e recuperação dos remanescentes de floresta. Neste sentido o conhecimento científico é fundamental para o embasamento de planos de manejo, sendo que no caso da Mata Atlântica, listagens de espécies muitas vezes são as únicas informações disponíveis. Apesar de comumente utilizarem métodos pouco comparáveis e esforços desiguais, essas listagens são valiosas para a compreensão dos padrões de distribuição das espécies auxiliando na elaboração de planos de conservação e manejo de áreas. Listagens de localidades ao longo das encostas da Serra do Mar podem ser encontradas em Willis \& Oniki (1981), Scott \& Brooke (1985), Höfling \& Lencioni (1992), Olmos (1996), Parker \& Goerck(1997), Aleixo \& Galleti (1997), Goerck (1999),
Vielliard \& Silva (2001) e Develey (2004a). Já nas regiões de planalto e interior, com matas mais alteradas, a quantidade de informação é menor, destacando-se os trabalhos de Anjos (2001a) e Anjos (2004). Também existe uma grande falta de informação referente a estimativas de abundância das espécies, sendo que muitas das listagens apresentam apenas resultados qualitativos.

Neste contexto, este trabalho vem preencher uma lacuna de informação sobre a composição e abundância da avifauna na região do Planalto Atlântico Paulista. Além do levantamento qualitativo e quantitativo das aves, esse artigo também compara dois dos principais métodos de amostragem, considerando a heterogeneidade da floresta dentro da área da Reserva.

\section{Material e Métodos}

\section{1.Área de Estudo}

A Reserva do Morro Grande possui uma área aproximada de 10870 ha e está situada no município de Cotia (2339‘S, $23^{\circ} 48^{\prime} \mathrm{S}$ e $\left.46^{\circ} 47^{\prime} \mathrm{W}, 4^{\circ} 55^{\prime} \mathrm{W}\right)$. Descrições detalhadas da Reserva e das localidades amostradas podem ser encontradas em Metzger et al. (2006). Foram amostradas cinco áreas diferentes dentro da Reserva sendo elas: Ruth (R), Área A (A), Área B (B), Área C (C) e Quilombo (Q). A área $\mathrm{R}, \mathrm{A}$ e $\mathrm{B}$ apresentavam vegetação mais alterada e $\mathrm{R}$ estava situada mais próxima à borda da Reserva. A área do Quilombo corresponde a um trecho de cerca de 60 hectares de vegetação primária em meio à vegetação secundária que predomina em toda a Reserva. Maiores detalhes sobre a estrutura e fisionomia da vegetação são apresentados em Metzger et al. (2006). Todas as áreas amostradas distavam entre si no mínimo $2 \mathrm{~km}$. Também foram realizadas observações esporádicas nas margens da represa situada no interior da Reserva e nas bordas da mata.

\section{Levantamento da Avifauna}

O levantamento geral das aves foi realizado entre os anos de 2001 e 2003. Foram utilizados dois métodos de amostragem diferentes: Pontos Fixos (Reynolds et al. 1980) e Redes de Neblina.

\subsection{Pontos Fixos}

Foram amostradas quatro áreas: A, B, C e Quilombo. Cinco pontos foram determinados em cada uma destas quatro áreas, sendo visitados cinco vezes cada um, totalizando 25 contagens por área e 100 contagens para toda a Reserva. Um mesmo ponto nunca foi visitado por mais de uma vez num único dia. Foi considerada uma distância mínima de 200 metros entre cada ponto. O tempo de amostragem em cada ponto foi de 10 minutos e as aves 
foram contadas considerando um raio máximo de detecção de aproximadamente 100 metros. As amostragens foram realizadas sempre nas três primeiras horas do dia, exclusivamente entre os meses de primavera e verão dos anos de 2001, 2002 e 2003. A abundância total das espécies foi calculada através da soma do número de contatos visuais ou aditivos obtidos nas quatro áreas amostradas.

\subsection{Redes de Neblina}

Foram utilizadas 10 redes de neblina (12 X 2,5 m, 36 $\mathrm{mm}$ ) armadas em linha contínua, abertas ao amanhecer (» 0600h) e fechadas seis horas depois (» 12:00 h), um dia por mês em cada área, entre os meses de março e novembro de 2001. O esforço total foi de 2147 horas/rede distribuídas eqüitativamente ao longo dos meses e das áreas (536,75 \pm 5,85 horas/rede por área). As redes foram abertas no nível do chão e as aves quando capturadas eram marcadas com anilhas numeradas padrão CEMAVE e soltas logo em seguida. Os seguintes locais foram amostrados com redes de neblina: Ruth (R), A, B e C. A soma do número total de capturas por espécie, excluíndo as recapturas de um mesmo indivíduo numa mesma manhã, foi utilizada como índice de abundância, de forma a tornar as comparações entre métodos mais fiéis, uma vez que através do método de ponto fixo fica impossível distinsões de indivíduos.

Além destes dois métodos quantitativos, também foram anotadas todas as espécies observadas durante o deslocamento entre as trilhas ou pontos de amostragem e o intervalo de checagem das redes. Desse modo, além dos dados quantitativos, também foi obtida uma lista qualitativa muito mais completa. Nesse caso, a maioria das espécies foi identificada através de observação direta com binóculos e muitas tiveram sua vocalização (incluindo gritos de alarme e chamadas curtas) gravada em fitas-cassete com gravador Sony TCM-5000 e microfone direcional Sennheiser ME-66.

As estimativas de abundância das dez espécies mais comuns através do método do ponto fixo foram comparadas com os resultados obtidos em outras duas áreas de Mata Atlântica no estado de São Paulo: o Parque Estadual de Intervales (41.705 ha), na Serra de Paranapiacaba (Vielliard \& Silva 2001) e a Estação Ecológica de Caetetus (2.178 ha), no município de Gália no interior do estado (Betini 2001). Estas duas localidades também foram amostradas através do método do ponto fixo e apesar de existirem variações no tempo e distância de amostragem, a ordenação de abundância das espécies pode ser comparada sem problemas.

A ordem sistemática e a nomenclatura das espécies de aves utilizadas nos apêndices, tabelas e gráficos seguem CBRO(2005).

\section{Análise dos Dados}

Com o objetivo de explorar os padrões de composição e abundância das espécies de aves foi utilizada uma análise de ordenação levando-se em conta os dois métodos de amostragem. Para tal utilizamos a Análise de Correspondência Destendenciada (DCA), no programa PC-ORD versão 4 (McCune \& Mefford, 1999). Para se verificar a existência de relação entre a abundância das espécies capturadas nas redes e as espécies amostradas nos pontos fixos, foi realizado um teste de correlação não paramétrico (Correlação de Spearman). Nesse teste foram consideradas apenas as espécies amostradas tanto nos pontos quanto nas redes.

\section{Resultados}

\section{Número de espécies}

Considerando todos os métodos de amostragem um total de 198 espécies foi encontrado na Reserva do Morro Grande, sendo 103 registradas por meio dos pontos fixos e redes de neblina, 98 (50\%) através dos pontos fixos e 46 (23\%) capturadas nas redes de neblina (Apêndice 1). Esses resultados indicam a seletividade e a limitação de ambos os métodos de amostragem quantitativa. Do total de espécies registradas 56 são endêmicas da Mata Atlântica, 149 foram observadas exclusivamente em ambientes florestais (incluindo as endêmicas) e outras 21 não são aves características de floresta e estavam presentes apenas nas bordas da mata ou na beira da represa. Onze espécies são consideradas globalmente ameaçadas ou quase-ameaçadas de extinção (BirdLife International 2000), sendo nove destas endêmicas do bioma Mata Atlântica (Stotz et al. 1996). Levando-se em conta o status de conservação das aves no estado de São Paulo (São Paulo 1998), 13 espécies presentes no Morro Grande estão ameaçadas.

Comparando-se as três áreas que foram amostradas através de pontos fixos e redes, o número de espécies registradas através do método do ponto fixo é superior em todas elas (Figura 1). Existe uma clara tendência, verificada por ambos os métodos, de aumento de riqueza, comparandose as áreas situadas mais próximas à borda da Reserva (R) ou em matas apresentando estádio sucessional mais novos (A, B), com a área central que apresenta estádio sucessional mais avançado (C). Além disso, o número de espécies registradas no Quilombo, área de mata primária, é o maior dentre todas as amostradas, apresentando 12 espécies exclusivas (Apêndice 1). A diferença entre a riqueza das cinco áreas amostradas e o número total de espécies registradas por redes ou pontos demonstram a grande heterogeneidade existente na comunidade de aves da Reserva. Do total de 98 espécies amostradas pelo método de ponto fixo, apenas 59 estavam presentes na área A, enquanto no Quilombo, a área mais rica da Reserva, 75 espécies foram registradas (Figura 1). Essa heterogeneidade também fica clara no resultado obtido na análise de ordenação (DCA) considerando a composição, abundância e métodos de amostragem (Figura 2). O primeiro eixo do gráfico (autovalor $=0,34$; porcentagem de explicação $=42 \%$ ) separou 
claramente os dois métodos utilizados, com os maiores valores no eixo correspondendo à amostragem com redes. Em relação ao segundo eixo (autovalor =0,08; porcentagem de explicação = $10 \%$ ), as áreas com maior riqueza apresentam os valores mais baixos (Quilombo no caso de pontos fixos e $\mathrm{B}$ e C no caso das redes).

\section{Abundância de espécies}

A espécie mais comum, segundo os dois métodos foi Chiroxiphia caudata, seguido nos pontos por Procnias nudicolis e Basileuterus leucoblepharus nas redes. Esta foi a terceira mais abundante nos pontos fixos (Apêndice 1). Algumas espécies com resultados discrepantes entre os métodos foram: Brotogeris tirica, Chamaeza campanisona, Attila rufus, Carpornis cucullatus, Myiobius barbatus e Trichothraupis melanops. Cinco espécies capturadas não foram amostradas nos pontos fixos (Drymophila ochrpyga, Cnemotriccus fuscatus, Myiobius barbatus, Turdus amaurochalinus e Haplospiza unicolor). Apesar destas diferenças existe uma correlação significativa entre a abundância das espécies capturadas nas redes e também amostradas nos pontos $\left(\mathrm{r}_{\mathrm{s}}=0,55 ; \mathrm{N}=41 ; \mathrm{p}<0,001\right)$.

Curiosamente, algumas espécies bastante comuns em locais particulares da Reserva não foram amostradas através de nenhum dos métodos quantitativos, como Polyborus plancus, uma espécie típica de áreas abertas que era observada com freqüência ao longo da estrada que corta o interior da Reserva.

A comparação das dez aves mais abundantes no Morro Grande, Intervales e Caetetus mostrou que apenas duas dessas estão entre as mais comuns nas três áreas: Sittasomus griseicapillus e Basileuterus culicivorus (Tabela 1). Entre esse conjunto de dez espécies no Morro Grande, cinco também são mais comuns em Intervales e três em Caetetus. A espécie mais abundante nesta última localidade (Herpsilochmus rufimarginatus) ocupa a décima primeira posição no Morro Grande, enquanto que em Intervales a espécie é bem mais rara. Outras espécies muito abundantes no Morro Grande e Intervales, como Procnias nudicollis e Chamaeza campanisona, estão ausentes ou são extremamente raras em Caetetus.

\section{Discussão}

A Reserva do Morro Grande apresenta um elevado número de espécies de aves florestais (149), no entanto esse valor é inferior ao registrado em outras áreas de Mata Atlântica. Na Estação Ecológica Juréia-Itatins, com 80.000 ha, um total de 194 espécies florestais foram encontradas (Develey 2004a). Nas encostas da Serra do Mar, no município de Ubatuba, 158 espécies são exclusivas de mata (Goerck 1999), enquanto que em Salesópolis o número de espécies florestais foi de 188 (Höfling \& Lencione 1992). No entanto, essas áreas apresentavam grandes extensões de matas primárias, além de um marcante gradiente altitudinal (Juréia e Ubatuba). No caso do Morro Grande, além do menor tamanho, a maior parte da mata já sofreu um intenso processo de perturbação, o que certamente levou a uma perda de espécies.

\section{Análise dos Métodos}

O número total de espécies amostradas é muito superior ao registrado pelo método de pontos fixos e redes. Essas diferenças também foram encontradas em outros levantamentos realizados em florestas tropicais, sendo que de maneira geral $60 \%$ da avifauna local é amostrada por pontos fixos (Whitman et al. 1997, Goerck 1999). No caso das redes de neblina a proporção é um pouco menor, sendo em média de 30 \% (Bierregaard Jr. 1990, Whitman et al. 1997).

Também existem diferenças comparando-se apenas os dois métodos quantitativos. Espécies de dossel, dificilmente são capturadas pelas redes de neblina, o que explica as diferenças encontradas no caso das famílias Psitacidae e Cotingidade. O comportamento das espécies também influencia o padrão de amostragem, como no caso de Myiobius barbatus, ave de sub-bosque, pouco conspícua e silenciosa, não sendo registrada nos pontos, mas capturada nas redes. Outro exemplo claro é Chamaeza campanisona, espécie terrestre que voa pouco, mas vocaliza com frequência, sendo raramente capturada nas redes e facilmente registrada nos pontos. Apesar de algumas espécies apresentarem diferenças marcantes em relação aos métodos, muitas mostram um padrão semelhante de abundância, como demonstrado na correlação entre as aves capturadas e amostradas nos pontos. Além disso, o padrão de distribuição das espécies nas diferentes áreas amostradas seguiu a mesma tendência nos dois métodos utilizados, mostrando que ambos são adequados e congruentes para amostragem de comunidades de aves florestais. O importante é se conhecer e respeitar as limitações de cada método. Apesar das dificuldades práticas, o ideal é que se utilizem os dois métodos de forma complementar, como no caso deste trabalho.

\section{Heterogeneidade da Avifauna}

Apesar da reduzida extensão de matas maduras na Reserva, existem claras diferenças entre estas áreas e as de matas secundárias com relação à avifauna. Das doze espécies presentes exclusivamente no Quilombo, dez são endêmicas e três enquadradas em alguma das categorias de ameaça. Por outro lado, espécies comuns nas áreas A, B e C não foram registradas no Quilombo, como Batara cinerea e Basileuterus leucoblepharus, ambas características de matas secundárias (Uezu 2002, Develey 2004b). Os trabalhos de Protomastro (2001) e Aleixo (1999) mostraram que matas secundárias podem abrigar uma alta diversidade de aves quando comparadas a matas primárias. No entanto, no caso do Morro Grande, além das diferenças na composição de aves entre áreas de mata primária e secundária, o número de 
espécies registrado no Quilombo também foi maior. Se a vegetação hoje presente nesta área é mesmo um testemunho do que predominava em toda a área da Reserva, muitas aves devem ter desaparecido. Esse empobrecimento da avifauna fica mais evidente considerando a severa perda de espécies da Reserva do Morro Grande em comparação com uma área de mata contínua primária na Serra de Paranapiacaba, a cerca de 40 km (Develey \& Metzger, no prelo). Com exceção da área do Quilombo, a avifauna típica de sub-bosque do Morro Grande é muito similar com a que é encontrada nos grandes fragmentos adjacentes (aprox. 100 ha, Martensen et al., no prelo). O mesmo não ocorre quando se considera as espécies de dossel, sendo que os pequenos papagaios, maitacas, tucanos e arapongas praticamente desapareceram dos fragmentos próximos (Develey 2004b) e em outras áreas de Mata Atlântica como no norte do Paraná (Anjos 2001a).

As diferenças de riqueza e composição da avifauna na Reserva, não ocorrem apenas entre as matas maduras e secundárias. Dentro das áreas R, A, B e C também existem diferenças, mostrando que mesmo matas secundárias apresentam diferenças na avifauna. Estas diferenças podem estar ligadas ao histórico de ocupação da área, que influenciam a regeneração da floresta, possivelmente afetando a comunidade de aves (Goerck 1995, Rahbek 1997, Borges \& Stouffer 1999).

\section{Padrões de abundância}

Em relação às dez aves mais comuns nas três áreas de Mata Atlântica consideradas, as diferenças encontradas entre esse conjunto de espécies estão ligadas a fatores como o tipo de mata, estado de conservação e localização. A vegetação da E. E. de Caetetus é classificada como floresta estacional semidecidual e a área está isolada de outras matas de tamanho significativo. Todas as espécies mais comuns são insetívoras, sendo que algumas são beneficiadas pelo processo de fragmentação, como Thamnophilus caerulescens e Conopophaga lineata (Anjos 2001b). Já no caso de Intervales e do Morro Grande as espécies mais comuns podem ser consideradas um pouco mais exigentes segundo Stotz et al. (1996). No entanto, os efeitos da perturbação na vegetação no Morro Grande determinam a alta abundância de Basileuterus leucoblepharus e Dysithamnus mentalis, espécies de mata secundária e comuns em fragmentos (Develey 2004b, Martensen et. al. no prelo). Apesar do Morro Grande estar localizado no planalto, sob certa influência das matas mais secas do interior, de modo geral sua avifauna é muito mais semelhante a áreas de matas úmidas situadas na Serra do Mar.

\section{Espécies raras ou localmente extintas}

Muitas aves encontradas em matas contínuas próximas ao Morro Grande não foram registradas na Reserva. No caso de alguns grupos, como as espécies cinegéticas, a forte pressão de caça na Reserva pode explicar tais ausências. O macuco (Tinamus solitarius) e a jacutinga (Pipile jacutinga) foram totalmente extintos em toda a Reserva, e mesmo o nhambu-guaçu (Crypturellus obsoletus) é muito raro. Outra espécie, ainda presente, mas difícil de ser vista é o jacu (Penelope obscura) que também já deve ter sido muito caçado. No caso de alguns frugívoros de dossel é mais difícil entender a ausência ou raridade das espécies na Reserva. Com exceção da araponga (Procnias nudicollis) e do corocochó (Carpornis cucullatus), todos os demais frugívoros são raros. Mesmo o corocochó (C. cucullatus) era freqüente apenas no Quilombo, e estava ausente nas áreas A e B. Espécies como Selenidera maculirostris e Lipaugus lanioides não foram registradas em nenhuma ocasião. O fato de grande parte da mata ser secundária deve influenciar a disponibilidade local de frutos. De acordo com Levey (1988), as matas secundárias apresentam uma variação sazonal na produção de frutos muito maior do que as matas primárias, de forma que devem ocorrer períodos de escassez de alimento. O palmito (Euterpe edulis) representa um recurso extremamente importante para muitas espécies frugívoras da Mata Atlântica (Galetti et al. 2000, Pizo et al. 2002) e foi praticamente extinto no Morro Grande, restando apenas na área do Quilombo. Mesmo nessa área a grande maioria dos indivíduos de $E$. edulis é composta por jovens que ainda não produzem frutos. Além de uma possível falta de recurso alimentar, outro fator que deve estar influenciando na raridade das espécies frugívoras é a falta de locais de nidificação, principalmente no caso dos Psitacídeos e Ramphastídeos. Essas aves nidificam em ocos de árvores (Sick 1997), recurso escasso em áreas de floresta, especialmente em matas secundárias, onde o porte das árvores é menor. Finalmente, características do relevo também podem explicar esse padrão de raridade dos frugívoros. Toda a Reserva é basicamente plana, não existindo locais com alta declividade ou vales encaixados. Na Mata Atlântica os fundos dos vales possuem árvores mais altas e maior riqueza e diversidade de espécies vegetais do que áreas situadas nos topos de morros (Mantovani et al. 1989). Desse modo, nesses locais deve haver uma maior disponibilidade de frutos e sítios de nidificação; a ausência desses vales no Morro Grande também pode ter levado a uma menor abundância e riqueza de aves frugívoras.

\section{Importância da Reserva e conservação}

Apesar de já ter passado por um intenso processo de perturbação, o que deve ter levado a perda de muitas espécies, a Reserva do Morro Grande ainda abriga uma comunidade de aves rica e diversa, com muitas espécies endêmicas e ameaçadas tanto a nível global quanto regional. O fato da Reserva estar situada próxima a matas contínuas e bem preservadas da Serra do Mar possibilitaria a colonização de algumas espécies que já desapareceram. No entanto, é fundamental que haja uma fiscalização na área impedindo 
atividades de caça, retirada ilegal de palmito e bromélias, além do desmatamento que ainda existe ao longo das bordas. Além disso, a longo prazo, outras medidas de manejo podem ser feitas, como o enriquecimento da vegetação já depauperada.

Um monitoramento contínuo da avifauna da Reserva seria extremamente importante para melhor compreender os padrões de distribuição das espécies. Os dados referentes aos tamanhos populacionais de muitas aves aqui apresentados são um importante ponto de partida para um monitoramento a longo prazo das aves da Reserva.

A singularidade da Reserva do Morro Grande, por encontrar-se em uma área relativamente plana e próxima à região metropolitana de São Paulo, deve ser levada em conta, tanto para sua proteção quanto para investigação. Geralmente as áreas que ainda se encontram cobertas por florestas na Mata Atlântica são extremamente íngremes, sendo que áreas extensas, planas e florestadas como a Reserva do Morro Grande são praticamente inexistentes, conferindo um maior valor para esta unidade de conservação.

\section{Agradecimentos}

A todos que propiciaram este estudo na RFMG, em particular a Rafael Pimentel pela sua enorme ajuda durante a amostragem e a José Roberto Nali e aos demais funcionários da SABESP na região, que sempre nos deram todo o apoio necessário para os trabalhos de campo. Este artigo foi beneficiado pelos excelentes comentários de dois assessores anônimos. Agradecemos a FAPESP pelas bolsas de doutorado e de iniciação científica concedidas aos autores. E finalmente gostaríamos de manifestar nossos sinceros agradecimentos a Jean Paul Metzger, que além de dar todo o suporte para os trabalhos de campo e de ler cuidadosamente uma versão inicial deste manuscrito, foi sempre um incentivador e orientador em nossos trabalhos.

\section{Referências bibliográficas}

ANJOS, L. 2001a. Bird communities in five Atlantic forest fragments in southern Brazil. Orn. Neot 12:11-27.

ANJOS, L. 2001b. Comunidade de aves florestais: implicações na conservação. In Ornitologia e Conservação: da ciência às estratégias (J.L.B. Albuquerque, J.F. Cândido Jr., F.C. Straube \& E.A. Roods, Eds.). Sociedade Brasileira de Ornitologia, Curitiba, Paraná, p.17-37.

ANJOS, L. 2004. Species richness and relative abundance of birds in natural and anthropogenic fragments of Brazilian Atlantic forest. Anais da Acad. Bras. Ciências 76:429-434.

ALEIXO, A. \& GALETTI, M. 1997. The conservation of the avifauna in a lowland Atlantic forest in south-east Brazil. Bird Cons. Inter 7:235-261.
ALEIXO, A. 1999. Effects of selective logging on a bird community in the Brazilian Atlantic Forest. Condor 101:537-548.

BETINI, G S. 2001. Amostragem de aves por pontos numa floresta estacional semidecidual, São Paulo, Brasil. Dissertação de mestrado, Universidade de São Paulo, Piracicaba.

BIERREGAARD Jr., R.O. 1990. Species composition and trophic organization of the understory bird community in a central Amazonian terra firme forest. In Four Neotropical Rainforests (A. H. Gentry, ed.). Yale University, New Haven.

BIRDLIFE INTERNATIONAL, 2000. Threatened birds of the world. Lynx Edicions and BirdLife International, Barcelona and Cambridge.

BORGES, S. H. \& STOUFFER, P. C. 1999. Bird communities in two types anthropogenics successional vegetation in Central Amazonia. Condor 101:529-536.

BROOKS, T. \& BALMFORD, A. 1996. Atlantic forest extinctions. Nature 380:115.

Comitê Brasileiro de Registros Ornitológicos (CBRO). 2005. http://www.cbro.org.br/CBRO/index.htm (último acesso em Janeiro 2006).

DEVELEY, P.F. 2004a. As aves da Estação Ecológica JuréiaItatins. In Estação Ecológica Juréia-Itatins - Ambiente físico, Flora e Fauna (Marques, O. A. V \& Duleba, W. eds). Ed Holos, Ribeirão Preto, p.278-295.

DEVELEY, P.F. 2004b. Efeitos da Fragmentação e do estado de Conservação da Floresta da diversidade de aves de Mata Atlântica. Tese de doutorado, Universidade de São Paulo, São Paulo.

DEVELEY, P.F. \& METZGER, J.P. Emerging threats to birds in Brazilian Atlantic Forest: the roles of forest loss and configuration in a severely fragmented ecosystem. In Emerging threats to tropical forests (C.A. Peres. \& W.F. Laurance, eds). University of Chicago Press, Chicago, no prelo.

GALETTI, M., LAPS, R. \& PIZO, M.A. 2000. Frugivory by toucans at two altitudes in Atlantic Forest of Brazil. Biotropica 32:842-850.

GOERCK, J.M. 1995. Birds of the Atlantic forest of Brazil: patterns of rarity and species distributions along an elevation gradient. Tese de mestrado, University of Missouri, St. Louis.

GOERCK, J.M. 1997. Patterns of rarity in the birds of the Atlantic forest of Brazil. Cons. Biol. 11:112-118.

GOERCK, J.M. 1999. Distribution of birds along an elevational gradient in the Atlantic forest of Brazil: implications for the conservation of endemic and endangered species. Bird Cons. Inter. 9:235-253.

HÖFLING, E. \& LENCIONI, F. 1992. Avifauna da floresta Atlântica, região de Salesópolis, Estado de São Paulo. Rev. Bras. Biol. 52: 361-378. 
LEVEY, D.J. 1988. Spatial and temporal variation in Costa Rican fruit and fruit-eating bird abundance. Ecol. Monog. 58: 251-269.

MANTOVANI, W., RODRIGUES, R.R., ROSSI, L., ROMANIUC-NETO, S., CATHARINO, E.L.M. \& CORDEIRO, I. 1989. A vegetação na Serra do Mar em Salesópolis, SP. In Anais do II Simpósio de ecossistemas da costa Sul e Sudeste brasileira: estrutura, funcionamento e manejo. São Paulo 2:348-384.

MARTENSEN, A.C., DEVELEY, P.F. \& METZGER, J.P. Effects of forest fragmentation on the understory bird community of the Atlantic Rainforest of Southern Brazil. Ornitologia Neotropical, no prelo.

McCUNE, B. \& MEFFORT, M.J. 1999. Multivariate analysis of ecological data, version 4.25, MjM Software. Gleneden Beach, Oregon.

METZGER, J.P. 2000. Tree functional group richness and landscape structure in a Brazilian tropical fragmented landscape. Ecol. Appl. 10:1147-1161.

METZGER, J.P., ALVES, L.A., CATHARINO, E.L.M., GOULART \& W., SIMÕES, S.J.C. 2006. Uma área de relevante interesse biológico, porém pouco conhecida: a Reserva Florestal do Morro Grande. Biota Neotrop. 6(2): http://www.biotaneotropica.org.br/ v6n2/pt/abstract?article+bn00406022006 (último acesso em 03/05/2006).

MYERS, N., MITTERMEIER, R.A., MITTERMEIER, C.G. FONSECA, G.A.B. \& KENT, J. 2000. Biodiversity hotspots for conservation priorities. Nature 403:853-858.

OLMOS, F. 1996. Missing species in São Sebastião island, southeastern Brazil. Pap. Avulsos Zool. 39: 329-349.

PARKER, T.A. III. \& GOERCK, J.M. 1997. The importance of national parks and biological reserves to bird conservation in the Atlantic forest region of Brazil. Ornithol. Monogr. 48:527-542.

PIZO, M.A., SILVA, W.R., GALETTI, M. \& LAPS, R. 2002. Frugivory in cotingas of the Atlantic Forest of Southeast Brazil. Ararajuba 10:177-185.

PROTOMASTRO, J.J. 2001. A test for preadaptation to human disturbances in the bird community of the Atlantic forest. In Ornitologia e conservação: da ciência às estratégias (J.L.B. Albuquerque, J.F. Cândido Jr., F.C. Straube \& E.A. Roods, Eds.). Sociedade Brasileira de Ornitologia, Curitiba, p.179-198.

RAHBEK, C. 1997. The relationship among area, elevation and regional species richness in neotropical birds. Am. Nat. 149:875-902.

REYNOLDS, R.T., SCOTT, J.M. \& NUSSBAUM, R.A. 1980. A variable circular-plot method for estimating bird numbers. Condor 82:309-313.

SÃO PAULO. 1998. Fauna ameaçada no Estado de São Paulo. Secretaria Estadual do Meio Ambiente/SEMASérie Probio, São Paulo.
SCOTT, D.A. \& BROOKE, M. de L. 1985. The endangered avifauna of Southeastern Brazil: a report on the BOU/ WWF expeditions of 1980/81 and 1981/82. In: Conservation of tropical forest birds. (A.W. Diamond \& T.E. Lovejoy, eds.). ICBP Technical Publications 4, International Council for Bird Preservation, Cambridge.

SICK, H. 1997. Ornitologia Brasileira. Ed. Nova Fronteira, Rio de Janeiro.

SECRETARIA DE ESTADO DE MEIO AMBIENTE (SMA). 1996. Atlas das unidades de conservação ambiental do Estado de São Paulo: parte I - Litoral. Cesp, São Paulo.

SOS MATA ATLÂNTICA/INPE 2002. Atlas da evolução dos remanescentes florestais da Mata Atlântica no período de 1995 - 2000. São Paulo.

STOTZ, D.F, FITZPATRICK, J.W., PARKER, T.A. III. \& MOSKOVITS, D.K. 1996. Neotropical birds: ecology and conservation. University of Chicago Press, Chicago.

UEZU, A. 2002. Efeito do tamanho de fragmentos e da conectividade na abundância de setes espécies de aves florestais em Caucaia do Alto, S.P. Dissertação de mestrado, Universidade de São Paulo, São Paulo.

VIANA, V.M. \& TABANEZ, A.J. 1996. Biology and conservation of forest fragments in the Brazilian Atlantic moist forest. In Forest patches in tropical landscapes (J. Schelhas \& R. Greenberg, eds.). Island Press, Washington, p 151-167.

VIELLIARD, J.M. \& SILVA, W.R. 2001. Avifauna. In Intervales (Leonel, C. ed.). Fundação para a conservação e produção florestal do Estado de São Paulo, São Paulo, p.123-139.

WILLIS, E.O. \& ONIKI, Y. 1981. Levantamento preliminar de aves em treze áreas do Estado de São Paulo. Rev. Bras. Biol. 41: 121-135.

WHITMAN, A.A., HAGAN III, J.M. \& BROKAW, V.L. 1997. A comparison of two bird survey techniques used in a subtropical forest. Condor 99: 955-965.

Título: Genus octacanthium (zygnemaphyceae, desmidiaceae) in the state of São Paulo, Brazil

Autores: Sílvia Maria Mathes Faustino \& Carlos E. de M. Bicudo

Biota Neotropica, Vol. 6 ( número 2 ): 2006

http://www.biotaneotropica.org.br/v6n2/pt/ abstract?article+bn00706022006

Recebido em 11/8/2004 - Versão reformulada recebida em 21/3/2005 - Publicado em 01/05/2006

ISSN 1676-0603 


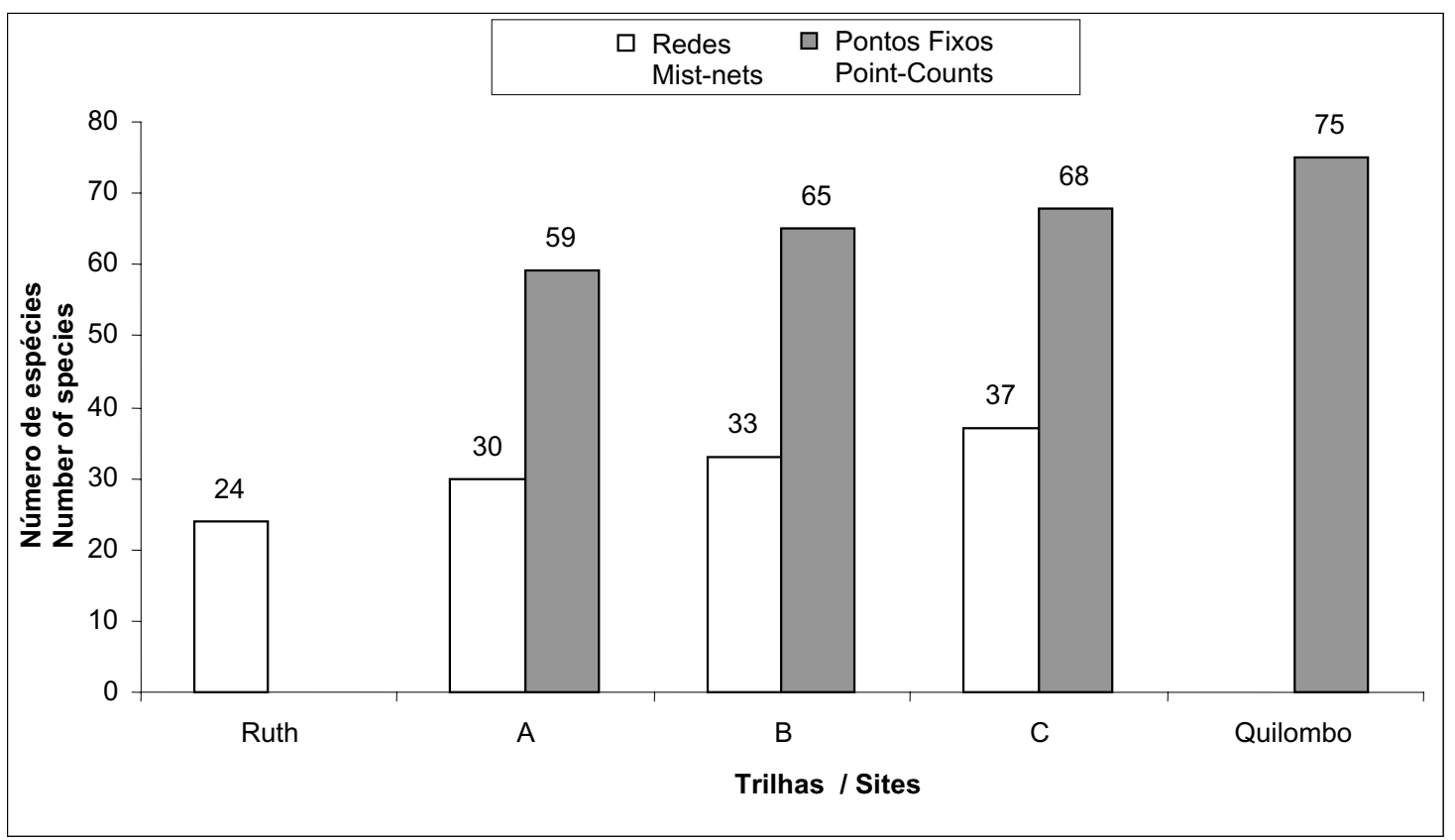

Figura 1. Número de espécies registradas através dos dois métodos de amostragem quantitativos empregados nas diferentes áreas da reserva do Morro Grande, Cotia, São Paulo, Brasil.

Figure 1. Number of species registered in each location with both quantitative methods in the Morro Grande Reserve, Cotia, São Paulo, Brazil. 


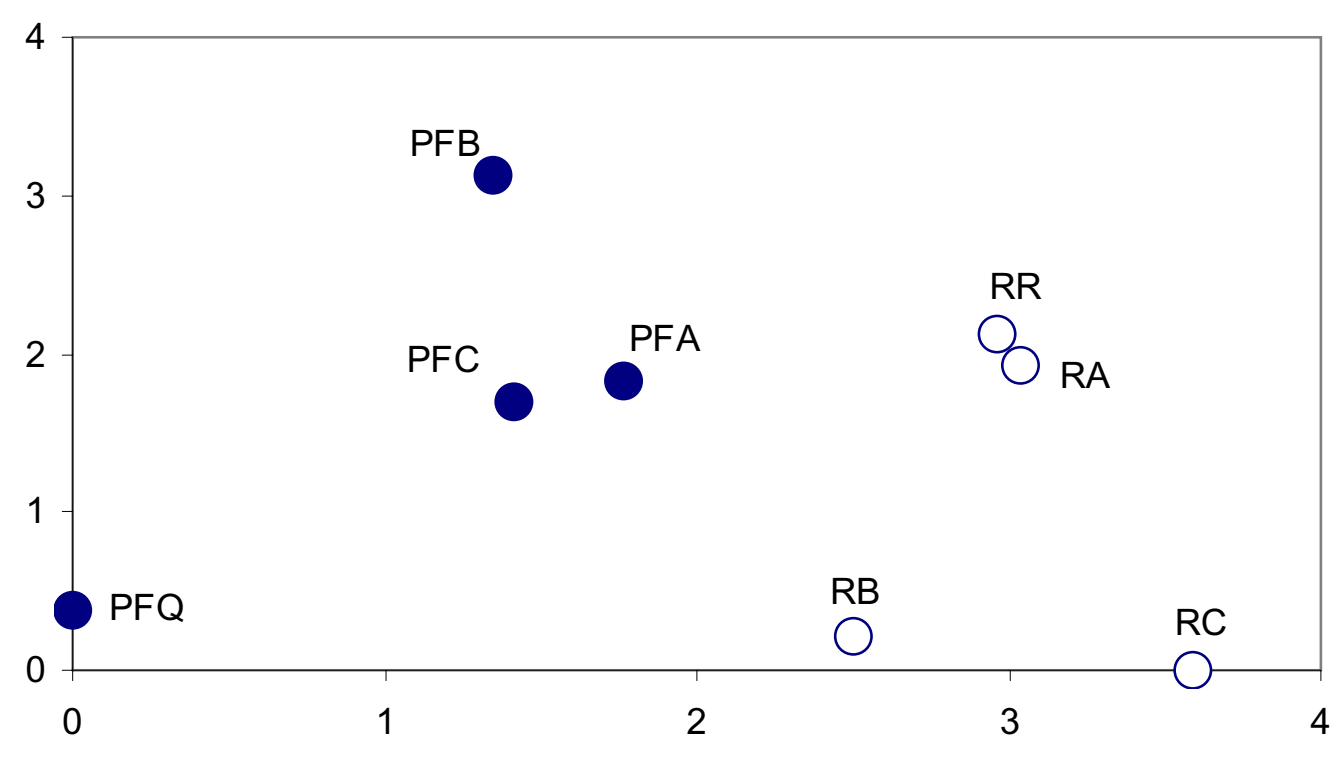

Figura 2. Ordenação das áreas amostradas na reserva através de uma Análise de Correspondência (DCA), com base na composição e abundância das aves amostradas pelo método das redes (R) e pontos fixos (PF). Áreas A, B, C, Ruth (R) e Quilombo (Q).

Figure 2. Ordination of the sampled sites by correspondence analyses, based on composition and abundance of the sampled species by both methods mist-nets and point-counts. Sites A, B, C, Ruth R and Quilombo (Q). 
Tabela 1. Dez espécies de aves mais comuns em três áreas de Mata Atlântica no estado de São Paulo. Espécies em ordem decrescente de abundância. Em negrito estão as espécies comuns nas três áreas.

\begin{tabular}{lll}
\hline \multicolumn{1}{c}{ Morro Grande (este estudo) } & \multicolumn{1}{c}{$\begin{array}{c}\text { Intervales (Vielliard \& } \\
\text { Silva 2001) }\end{array}$} & \multicolumn{1}{c}{ Caetetus (Betini 2001) } \\
\hline $1^{\circ}$ ) Chiroxiphia caudata & $1^{\circ}$ ) Chamaeza campanisona & $1^{\circ}$ ) Basileuterus culicivorus \\
$2^{\circ}$ ) Procnias nudicollis & $2^{\circ}$ ) Anabazenops fuscus & $2^{\circ}$ ) Herpsilochmus rufimarginatus \\
$3^{\circ}$ ) Basileuterus leucoblepharus & $3^{\circ}$ ) Trogon viridis & $3^{\circ}$ ) Vireo olivaceus \\
$4^{\circ}$ Sittasomus griseicapillus & $4^{\circ}$ Sittasomus griseicapillus & $4^{\circ}$ ) Thamnophilus caerulescens \\
$5^{\circ}$ ) Chamaeza campanisona & $5^{\circ}$ ) Chiroxiphia caudata & $5^{\circ}$ ) Dysithamnus mentalis \\
$6^{\circ}$ Carpornis cucullata & $6^{\circ}$ Procnias nudicollis & $6^{\circ}$ ) Hemitriccus orbitatus \\
$7^{\circ}$ ) Schiffornis virescens & $7^{\circ}$ ) Patagioenas plumbea & $7^{\circ}$ Sittasomus griseicapillus \\
$8^{\circ}$ ) Trogon surrucura & $8^{\circ}$ Pyriglena leucoptera & $8^{\circ}$ ) Lathrotriccus euleri \\
$9^{\circ}$ Basileuterus culicivorus & $9^{\circ}$ ) Brotogeris tirica & $9^{\circ}$ ) Conopophaga lineata \\
$\left.1^{\circ}\right)$ Dysithamnus mentalis & $1^{\circ}$ ) Basileuterus culicivorus & $\left.1^{\circ}\right)$ Drymophila ferruginea \\
\hline
\end{tabular}

http://www.biotaneotropica.org.br 
Apêndice 1. Composição e abundância obtida através dos dois métodos de amostragem na reserva do Morro Grande, São Paulo, Cotia, Brasil. Apendice 1. Species composition and abundance obtained with the two employed method of sampling in the Morro Grande reserve, São Paulo, Cotia, Brazil.

\begin{tabular}{|c|c|c|c|c|c|}
\hline \multirow[b]{2}{*}{ Família e Espécie } & \multicolumn{2}{|c|}{$\begin{array}{l}\text { Abundância } \\
\text { Abundance }\end{array}$} & \multirow[t]{3}{*}{$\begin{array}{l}\text { Habitat } \\
\text { Habitat }\end{array}$} & \multicolumn{2}{|c|}{$\begin{array}{c}\text { Grau de Ameaça } \\
\text { Conservation Status }\end{array}$} \\
\hline & $\begin{array}{l}\text { Pontos } \\
\text { Fixos }\end{array}$ & Redes & & Global & Estado de SP \\
\hline Family and Species & $\begin{array}{l}\text { Point } \\
\text { counts }\end{array}$ & $\begin{array}{l}\text { Mist } \\
\text { nets }\end{array}$ & & Global & $\begin{array}{l}\text { State of São } \\
\text { Paulo }\end{array}$ \\
\hline \multicolumn{6}{|l|}{ TINAMIDAE } \\
\hline $\begin{array}{l}\text { Crypturellus obsoletus } \\
\text { Crypturellus tataupa } \\
\text { ANATIDAE }\end{array}$ & 3 & & $\begin{array}{l}\text { Ms } \\
\mathrm{Ms}\end{array}$ & & \\
\hline $\begin{array}{l}\text { Amazonetta brasiliensis } \\
\text { CRACIDAE }\end{array}$ & & & $\mathrm{L}$ & & \\
\hline $\begin{array}{l}\text { Penelope obscura } \\
\text { ODONTOPHORIDAE }\end{array}$ & & & Ms, Mm & & PA \\
\hline $\begin{array}{l}\text { Odontophorus capueira - End } \\
\text { PODICEPIDAE }\end{array}$ & 4 & & Ms, Mm & & \\
\hline $\begin{array}{l}\text { Podilymbus podiceps } \\
\text { PHALACROCORACIDAE }\end{array}$ & & & $\mathrm{L}$ & & \\
\hline $\begin{array}{l}\text { Phalacrocorax brasilianus } \\
\text { ARDEIDAE }\end{array}$ & & & $\mathrm{L}$ & & \\
\hline Butorides striata & & & $\mathrm{L}$ & & \\
\hline Ardea cocoi & & & $\mathrm{L}$ & & \\
\hline Ardea alba & & & $\mathrm{L}$ & & \\
\hline Egretta thula & & & $\mathrm{L}$ & & \\
\hline THRESKIORNITHIDAE & & & & & \\
\hline $\begin{array}{l}\text { Platalea ajaja } \\
\text { CATHARTIDAE }\end{array}$ & & & $\mathrm{L}$ & & \\
\hline Cathartes aura & & & Ms & & \\
\hline $\begin{array}{l}\text { Coragyps atratus } \\
\text { PANDIONIDAE }\end{array}$ & & & Ms & & \\
\hline $\begin{array}{l}\text { Pandion haliaetus } \\
\text { ACCIPITRIDAE }\end{array}$ & & & $\mathrm{L}$ & & $\mathrm{PA}$ \\
\hline Leptodon cayanensis & & & $\mathrm{Ms}, \mathrm{Mm}$ & & $\mathrm{PA}$ \\
\hline Elanus leucurus & & & $\mathrm{Al}$ & & \\
\hline Leucopternis lacernulata - End & & & Ms & VU & $\mathrm{CP}$ \\
\hline Rupornis magnirostris & & & $\mathrm{Ms}, \mathrm{Al}$ & & \\
\hline Buteo brachyurus & & & Ms & & \\
\hline Spizaetus tyrannus & & & Ms & & VU \\
\hline FALCONIDAE & & & & & \\
\hline Caracara plancus & & & $\mathrm{Ms}, \mathrm{Al}$ & & \\
\hline Milvago chimachima & & & $\mathrm{Ms}, \mathrm{Al}$ & & \\
\hline Herpetotheres cachinnans & & & Ms & & \\
\hline Micrastur ruficollis & 9 & 4 & $\mathrm{Mm}, \mathrm{Ms}$ & & \\
\hline $\begin{array}{l}\text { Micrastur semitorquatus } \\
\text { RALLIDAE }\end{array}$ & 3 & & Ms & & \\
\hline
\end{tabular}


Aramides saracura - End.

JACANIDAE

Jacana jacana

CHARADRIIDAE

Vanellus chilensis

COLUMBIDAE

Columbina talpacoti

Columba livia

Patagioenas cayennensis

Patagioenas plumbea

Leptotila verreauxi

Leptotila rufaxilla

Geotrygon montana

PSITTACIDAE

Pyrrhura frontalis - End

Forpus xanthopterygius

Brotogeris tirica-End

Pionopsitta pileata-End

Pionus maximiliani

Triclaria malachitacea-End

CUCULIDAE

Piaya cayana

Crotophaga ani

Guira guira

STRIGIDAE

Megascops choliba

Pulsatrix koeniswaldiana-End

Athene cunicularia

NYCTIBIIDAE

Nyctibius griseus

CAPRIMULGIDAE

Lurocalis semitorquatus

Nyctidromus albicollis

Macropsalis forcipata

APODIDAE

Streptoprocne zonaris

Chaetura cinereiventris

TROCHILIDAE

Phaethornis eurynome- End

Eupetomena macroura

Florisuga fusca-End

Thalurania glaucopis-End

Amazilia versicolor

Amazilia lactea

TROGONIDAE

Trogon surrucura-End

Trogon rufus

ALCEDINIDAE

Ceryle torquatus

Chloroceryle amazona

Chloroceryle americana

BUCCONIDAE

Malacoptila striata

RAMPHASTIDAE

Ramphastos dicolorus-End PICIDAE
Ms, L

L

$\mathrm{L}, \mathrm{Al}$

Al

$\mathrm{Al}$

Ms

Ms, Mm

Ms

Ms

Ms

Ms, Mm

$\mathrm{Ms}, \mathrm{Al}$

$\mathrm{Ms}, \mathrm{Mm}$

$\mathrm{Ms}, \mathrm{Mm}$

$\mathrm{Ms}, \mathrm{Mm}$

$\mathrm{Ms}, \mathrm{Mm}$

VU

VU

12

4

3

Ms

Al

Al

Ms

Ms

Al

Ms

Ms

Ms

Ms

Ms

Ms

$11 \quad 19 \quad$ Ms, Mm

$\mathrm{Al}$

Ms, Mm

$169 \mathrm{Ms}, \mathrm{Mm}$

1 Ms

Ms

$39 \quad \mathrm{Ms}, \mathrm{Mm}$

$10 \quad 1 \quad \mathrm{Ms}, \mathrm{Mm}$

L

L

$\mathrm{L}$

Ms, Mm

http://www.biotaneotropica.org.br 
Picumnus cirratus

3

Veniliornis spilogaster- End

Piculus aurulentus-End

Colaptes campestris

Celeus flavescens

Dryocopus lineatus

THAMNOPHILIDAE

Hypoedaleus guttatus- End

Batara cinerea

Thamnophilus caerulescens

Dysithamnus stictothorax-End

Dysithamnus mentalis

Myrmotherula gularis-End

Herpsilochmus rufimarginatus

Drymophila ochropyga-End

Drymophila malura - End

Terenura maculata-End

Pyriglena leucoptera-End

Myrmeciza squamosa-End

CONOPOPHAGIDAE

Conopophaga lineata-End

GRALLARIIDAE

Grallaria varia

RHINOCRYPTIDAE

Scytalopus indigoticus

FORMICARIIDAE

Chamaeza campanisona

Chamaeza meruloides-End

SCLERURIDAE

Sclerurus scansor- End

DENDROCOLAPTIDAE

Dendrocincla turdina - End

Sittasomus griseicapillus

Xiphocolaptes albicollis

Dendrocolaptes platyrostris

Xiphorhynchus fuscus-End

Lepidocolaptes squamatus - End

FURNARIIDAE

Furnarius rufus

Synallaxis ruficapilla- End

Synallaxis spixi

Cranioleuca pallida

Anabacerthia amaurotis-End

Syndactyla rufosuperciliata-End

Philydor atricapillus-End

Philydor rufum

Cichlocolaptes leucophrus-End

Automolus leucophthalmus-End

Lochmias nematura

Heliobletus contaminatus - End

Xenops minutus

Xenops rutilans

TYRANNIDAE

Mionectes rufiventris-End

Leptopogon amaurocephalus

Hemitriccus diops - End

\begin{tabular}{|c|c|c|}
\hline 5 & \multirow{2}{*}{1} & $\mathrm{Ms}, \mathrm{Mm}$ \\
\hline 9 & & $\mathrm{Ms}, \mathrm{Mm}$ \\
\hline 4 & & $\mathrm{Ms}, \mathrm{Mm}$ \\
\hline & & $\mathrm{Al}$ \\
\hline & & Ms \\
\hline 4 & & Ms \\
\hline 1 & & Ms \\
\hline 5 & & $\mathrm{Ms}$ \\
\hline 16 & 2 & Ms \\
\hline 1 & & $\mathrm{Mm}$ \\
\hline 32 & 15 & Ms, $\mathrm{Mm}$ \\
\hline 3 & & $\mathrm{Mm}$ \\
\hline 24 & & $\mathrm{Ms}, \mathrm{Mm}$ \\
\hline & 1 & $\mathrm{Ms}$ \\
\hline & & Ms \\
\hline & & $\mathrm{Mm}$ \\
\hline 10 & 24 & $\mathrm{Ms}, \mathrm{Mm}$ \\
\hline 3 & 2 & $\mathrm{Ms}, \mathrm{Mm}$ \\
\hline 10 & 18 & Ms \\
\hline 29 & & $\mathrm{Ms}, \mathrm{Mm}$ \\
\hline & & $\mathrm{Ms}, \mathrm{Mm}$ \\
\hline 48 & 3 & $\mathrm{Ms}, \mathrm{Mm}$ \\
\hline 1 & & $\mathrm{Ms}$ \\
\hline 15 & 18 & $\mathrm{Ms}, \mathrm{Mm}$ \\
\hline 1 & & $\mathrm{Mm}$ \\
\hline 60 & 42 & $\mathrm{Ms}, \mathrm{Mm}$ \\
\hline 7 & & $\mathrm{Ms}, \mathrm{Mm}$ \\
\hline 3 & 3 & $\mathrm{Ms}$ \\
\hline 52 & 29 & $\begin{array}{l}\mathrm{Ms}, \mathrm{Mm} \\
\mathrm{Mm}\end{array}$ \\
\hline
\end{tabular}

NT

NT

NT
Al

$\mathrm{Ms}$

Al

Ms

$\mathrm{Mm}$

$5 \quad$ Ms

8 Ms, Mm

$4 \quad \mathrm{Ms}, \mathrm{Mm}$

$\mathrm{Mm}, \mathrm{Ms}$

Ms

$1 \mathrm{Ms}$

Ms

$4 \quad \mathrm{Ms}, \mathrm{Mm}$

$\mathrm{Ms}$

$4 \quad 4 \quad \mathrm{Ms}, \mathrm{Mm}$

$9 \quad 3 \quad \mathrm{Ms}, \mathrm{Mm}$

$1613 \mathrm{Ms}, \mathrm{Mm}$

http://www.biotaneotropica.org.br 


\begin{tabular}{|c|c|c|c|c|c|}
\hline Hemitriccus orbitatus - End & 5 & 13 & Ms, Mm & NT & \\
\hline Poecilotriccus plumbeiceps & & & Ms & & \\
\hline Phyllomyias burmeisteri & & & $\mathrm{Mm}$ & & \\
\hline Phyllomyias fasciatus & 4 & & $\mathrm{Mm}$ & & \\
\hline Elaenia flavogaster & & & Ms & & \\
\hline Elaenia mesoleuca & 1 & & Ms & & \\
\hline Camptostoma obsoletum & 7 & & Ms & & \\
\hline Phylloscartes ventralis & & & Ms & & \\
\hline Myiornis auricularis - End & 4 & & Ms & & \\
\hline Tolmomyias sulphurescens & 13 & 2 & $\mathrm{Ms}, \mathrm{Mm}$ & & \\
\hline Platyrinchus mystaceus & 15 & 34 & Ms & & \\
\hline Platyrinchus leucoryphus & 1 & & $\mathrm{Mm}$ & NT & EP \\
\hline Myiobius barbatus & & 8 & Ms & & \\
\hline Lathrotriccus euleri & 12 & & Ms & & \\
\hline Cnemotriccus fuscatus & & 4 & Ms & & \\
\hline Colonia colonus & & & Ms & & \\
\hline Legatus leucophaius & 2 & & Ms & & \\
\hline Myiozetetes similis & & & $\mathrm{Al}, \mathrm{Ms}$ & & \\
\hline Pitangus sulphuratus & & & $\mathrm{Ms}, \mathrm{Al}$ & & \\
\hline Conopias trivirgata & & & $\mathrm{Ms}$ & & \\
\hline Myiodynastes maculatus & 5 & & Ms & & \\
\hline Megarynchus pitangua & & & $\mathrm{Ms}, \mathrm{Al}$ & & \\
\hline Empidonomus varius & & & $\mathrm{Al}$ & & \\
\hline Tyrannus melancholicus & & & $\mathrm{Al}$ & & \\
\hline Tyrannus savana & & & $\mathrm{Al}$ & & \\
\hline Sirystes sibilator & & & Ms, Mm & & \\
\hline Myiarchus swainsoni & 6 & & Ms & & \\
\hline Myiarchus ferox & & & $\mathrm{Al}$ & & \\
\hline Attila phoenicurus & 18 & & Ms, Mm & & \\
\hline $\begin{array}{l}\text { Attila rufus-End } \\
\text { COTINGIDAE }\end{array}$ & 21 & 1 & $\mathrm{Ms}, \mathrm{Mm}$ & & \\
\hline Carpornis cucullata - End & 47 & & Ms, Mm & NT & \\
\hline Procnias nudicollis-End & 80 & & $\mathrm{Ms}, \mathrm{Mm}$ & NT & VU \\
\hline $\begin{array}{l}\text { Pyroderus scutatus-End } \\
\text { PIPRIDAE }\end{array}$ & 6 & & $\mathrm{Ms}, \mathrm{Mm}$ & & EP \\
\hline Neopelma chrysolophum-End & 5 & 7 & Ms & & \\
\hline $\begin{array}{l}\text { Chiroxiphia caudata- End } \\
\text { TITYRIDAE }\end{array}$ & 82 & 76 & $\mathrm{Ms}, \mathrm{Mm}$ & & \\
\hline Schiffornis virescens-End & 44 & 26 & Ms, Mm & & \\
\hline Laniisoma elegans* & & & $\mathrm{Ms}$ & VU & VU \\
\hline Tityra cayana & 2 & & Ms, Mm & & \\
\hline Pachyramphus castaneus & & & $\mathrm{Ms}, \mathrm{Mm}$ & & \\
\hline Pachyramphus polychopterus & 10 & & Ms & & \\
\hline Pachyramphus marginatus & 1 & & $\mathrm{Mm}$ & & PA \\
\hline Pachyramphus validus & & & $\mathrm{Al}$ & & \\
\hline VIREONIDAE & & & & & \\
\hline Cyclarhis gujanensis & 18 & & Ms & & \\
\hline Vireo olivaceus & 31 & & Ms & & \\
\hline $\begin{array}{l}\text { Hylophilus poicilotis - End } \\
\text { HIRUNDINIDAE }\end{array}$ & 28 & & Ms, Mm & & \\
\hline Progne chalybea & & & $\mathrm{Ms}, \mathrm{Al}$ & & \\
\hline Pygochelidon cyanoleuca & & & $\mathrm{Ms}, \mathrm{Al}$ & & \\
\hline Stelgidopteryx ruficollis & & & $\mathrm{Ms}, \mathrm{Al}$ & & \\
\hline
\end{tabular}

TROGLODYTIDAE

Troglodytes musculus

Al TURDIDAE 


\begin{tabular}{|c|c|c|c|c|c|}
\hline Platycichla flavipes & 21 & 9 & Ms, Mm & & \\
\hline Turdus rufiventris & 26 & 13 & $\mathrm{Ms}, \mathrm{Al}$ & & \\
\hline Turdus amaurochalinus & & 3 & $\mathrm{Al}$ & & \\
\hline Turdus albicollis & 29 & 49 & Ms, Mm & & \\
\hline \multicolumn{6}{|l|}{ COEREBIDAE } \\
\hline Coereba flaveola & 5 & & Ms, Mm & & \\
\hline \multicolumn{6}{|l|}{ THRAUPIDAE } \\
\hline Orthogonys chloricterus - End & & & $\mathrm{Mm}$ & & \\
\hline Thlypopsis sordida & & & Ms & & \\
\hline Trichothraupis melanops & 6 & 52 & Ms, Mm & & \\
\hline Habia rubica & 7 & 2 & $\mathrm{Ms}, \mathrm{Mm}$ & & \\
\hline Tachyphonus cristatus & & & $\mathrm{Ms}, \mathrm{Mm}$ & & \\
\hline Tachyphonus coronatus - End & 3 & 11 & $\mathrm{Ms}, \mathrm{Mm}$ & & \\
\hline Thraupis sayaca & 1 & & $\mathrm{Ms}, \mathrm{Mm}$ & & \\
\hline Thraupis cyanoptera & 6 & & Ms, Mm & NT & \\
\hline Thraupis ornata & & & $\mathrm{Ms}, \mathrm{Mm}$ & & \\
\hline Thraupis palmarum & & & Ms, Mm & & \\
\hline Pipraeidea melanonota & & & $\mathrm{Ms}, \mathrm{Mm}$ & & \\
\hline Tangara seledon-End & 2 & & $\mathrm{Mm}$ & & \\
\hline Tangara desmaresti-End & & & $\mathrm{Ms}, \mathrm{Mm}$ & & \\
\hline Tangara preciosa & & & $\mathrm{Ms}$ & & PA \\
\hline Tersina viridis & & & Ms & & \\
\hline Dacnis cayana & & & Ms, Mm & & \\
\hline Hemithraupis ruficapilla-End & & & $\mathrm{Ms}, \mathrm{Mm}$ & & \\
\hline Conirostrum speciosum & & & Ms & & \\
\hline \multicolumn{6}{|l|}{ EMBERIZIDAE } \\
\hline Zonotrichia capensis & & & $\mathrm{Al}$ & & \\
\hline Haplospiza unicolor-End & & 9 & Ms & & \\
\hline Volatinia jacarina & & & $\mathrm{Al}$ & & \\
\hline Sporophila caerulescens & & & $\mathrm{Al}$ & & \\
\hline \multicolumn{6}{|l|}{ CARDINALIDAE } \\
\hline Saltator similis & 7 & 7 & Ms & & \\
\hline \multicolumn{6}{|l|}{ PARULIDAE } \\
\hline Parula pitiayumi & 24 & & Ms, Mm & & \\
\hline Geothlypis aequinoctialis & & & $\mathrm{Al}$ & & \\
\hline Basileuterus culicivorus & 35 & 4 & Ms, Mm & & \\
\hline $\begin{array}{l}\text { Basileuterus leucoblepharus-End } \\
\text { ICTERIDAE }\end{array}$ & 67 & 69 & Ms & & \\
\hline Cacicus chrysopterus & & & Ms, Mm & & \\
\hline \multicolumn{6}{|l|}{ FRINGILLIDAE } \\
\hline Euphonia violacea & & & Ms, Mm & & \\
\hline Euphonia pectoralis-End & 11 & & $\mathrm{Ms}, \mathrm{Mm}$ & & \\
\hline
\end{tabular}

Total: 198 espécies. End. - Espécies endêmicas da Mata Atlântica.

Total of: 198 species. End - Endemic from the Atlantic Rainforest
Abundância:
Pontos Fixos - número total de contatos para cada espécie
Redes - número total de capturas de cada espécie.
Point-Counts - total number of records
Mist-nets - total number of captures 


\section{Hábitat:}

Mm- Mata madura; Ms- Mata secundária; Al- áreas alteradas nas bordas da Reserva; L Lago

Mm- old-growth forest; Ms - Secondary forest; Al - altered habitat; L - Reservoir;

\section{Grau de Ameaça:}

Globalmente / Globally - BirdLife International 2000

VU - Vulnerável (vulnerable)

NT- quase ameaçadas ("near threatened")

Estado de São Paulo (SEMA) - State of São Paulo

VU - Vulnerável (vulnerable)

EP - Em Perigo (near threatened)

PA - Provavelmente ameaçado (probably threatened) 